\title{
Diphoton Background to Higgs Boson Production at the LHC with Soft Gluon Effects
}

\author{
C. Balázs, P. Nadolsky, C. Schmidt, and C.-P. Yuan \\ Michigan State University, East Lansing, MI 48824, U.S.A.
}

(June 1, 1999)

\begin{abstract}
The detection and the measurement of the production cross section of a light Higgs boson at the CERN Large Hadron Collider demand the accurate prediction of the background distributions of photon pairs. To improve this theoretical prediction, we present the soft-gluon resummed calculation of the $p p \rightarrow \gamma \gamma X$ cross section, including the exact one loop $g g \rightarrow \gamma \gamma g$ contribution. By incorporating the most important higher order contributions, the resummed cross section provides a reliable prediction for the inclusive diphoton invariant mass and transverse momentum distributions. Given our results, we propose the search for the Higgs boson in the inclusive diphoton mode with a cut on the transverse momentum of the photon pair, without the requirement of an additional tagged jet.
\end{abstract}

PACS number(s): 12.38.Cy, 13.85.Qk.

hep-ph/9905551, CTEQ-905, MSUHEP-90526

\section{INTRODUCTION}

The direct search for the Standard Model (SM) Higgs boson at the CERN LEP collider constrains its mass $m_{H}$ to be greater than $\sqrt{S}-m_{Z} \sim 95 \mathrm{GeV}$ [1]. Meanwhile, recent electroweak global fits [2], and the measurements of the $W^{ \pm}$boson and top quark masses [3] suggest that the SM Higgs mass is lower than about $260 \mathrm{GeV}$. If $m_{H}$ is less than twice the $Z^{0}$ boson mass, as the electroweak fits hint, then at the CERN Large Hadron Collider (LHC) the most promising detection modes of the SM Higgs boson will be $p p \rightarrow H X \rightarrow \gamma \gamma X[4]$ and the associated production $p p \rightarrow H$ jet $X \rightarrow \gamma \gamma$ jet $X$ [5]. According to earlier studies, a statistical significance on the order of 510 can be reached for both of these signals, actual values depending on luminosity and background estimates. In Ref. [5] it was also found that in order to optimize the significance it is necessary to impose a $30 \mathrm{GeV}$ cut on the transverse momentum of the jet, or equivalently (at NLO precision), on the $Q_{T}$ of the photon pair. With this cut in place extraction of the signal in the Higgs plus jet mode requires the precise knowledge of both the signal and background distributions in the mid- to high- $Q_{T}$ region.

The precise determination of signal and background rates of the inclusive diphoton process demands the calculation of the large QCD corrections. Higher order corrections, both to the signal [6] and the background [7], increase the rate significantly, by a factor of about 2 . In the case of the background this large increase is mostly due to the fact that the diphoton cross section receives a large contribution from the formally higher order $g g \rightarrow \gamma \gamma$ partonic subprocess, which is of the same order of magnitude as the $q \bar{q}+q g \rightarrow \gamma \gamma X$ subprocesses [8]. Since the lowest order $g g \rightarrow \gamma \gamma$ subprocess proceeds through a box diagram, the calculation of yet higher order corrections to this process is complicated.

A reliable calculation of the transverse momentum dis- tribution of the photon pair also requires the resummation of the potentially large logarithmic contributions of the type $\ln \left(Q / Q_{T}\right)$ (where $Q$ is the invariant mass of the pair), arising as a result of multiple soft-gluon emission. Using the soft-gluon resummation technique, the low- to mid- $Q_{T}$ region can be predicted, and the resummed calculation can be matched to the fixed order, giving a reliable prediction in the whole $Q_{T}$ range [8]. As an added bonus the resummed cross section also exhibits reduced scale dependence since it includes the most important higher order contributions. It also gives a hint of the size of the yet higher order corrections.

When $Q_{T}$ is integrated, the resummed calculation of Ref.[8] gives the rates for the $q \bar{q}+q g \rightarrow \gamma \gamma X$ subprocesses at the $\mathcal{O}\left(\alpha^{2} \alpha_{S}\right)$ precision. That calculation also includes the photon fragmentation contribution, and approximates the $\mathcal{O}\left(\alpha^{2} \alpha_{S}^{3}\right) g g+q g \rightarrow \gamma \gamma X$ rate. In this work, we include the exact one loop $\mathcal{O}\left(\alpha^{2} \alpha_{S}^{3}\right) g g \rightarrow \gamma \gamma g$ calculation to improve the diphoton background prediction in the high $Q_{T}$ region. The above fixed order and resummed contributions are implemented in the ResBos [9] Monte Carlo event generator which produces our numerical results.

\section{ANALYTICAL RESULTS}

The one loop $g g \rightarrow \gamma \gamma g$ amplitude can easily be derived from the one loop five-gluon $(5 g)$ amplitudes of Ref. [11] for the case when the particles in the loop are fermions in the fundamental representation, by replacing two of the gluon vertices with photon vertices. Since the $5 g$ amplitude is explicitly given in a color-decomposed form, it is possible to replace the $\mathrm{SU}(3)$ generators and strong couplings $\left(g_{S}\right)$ of two of the gluons with $\mathrm{U}(1)$ generators and the electromagnetic couplings $(e)$ of photons. The final expression for the square of the three-gluontwo-photon $(3 g 2 \gamma)$ amplitude is 


$$
\begin{gathered}
\left|A\left(g_{1} g_{2} \rightarrow g_{3} \gamma_{4} \gamma_{5}\right)\right|^{2}=8\left(e Q_{i}\right)^{4} g_{S}^{6} N_{C}\left(N_{C}^{2}-1\right) \times \\
\sum_{\text {helicities }}\left|\sum_{\sigma \in \mathrm{COP}_{4}^{(123)}} A_{5,1}^{[1 / 2]}\left(\sigma_{1}, \sigma_{2}, \sigma_{3}, \sigma_{4}, \sigma_{5}\right)\right|^{2},
\end{gathered}
$$

where $\sigma_{i}$ is shorthand for the 4-momenta and helicities, $\left\{p_{\sigma_{i}}, \lambda_{\sigma_{i}}\right\}$, of the gluons $1,2,3$ and the photons 4,5 . The charge of the quarks in the loop is given by $Q_{i}$ in the units of the charge of the positron, and $N_{C}=3$ is the number of colors in QCD. In Eq.(1) $\mathrm{COP}_{4}^{(123)}$ denotes the subset of permutations of $(1,2,3,4)$ that leave the ordering of $(1,2,3)$ unchanged up to a cyclic permutation

$$
\begin{gathered}
\sum_{\sigma \in \mathrm{COP}_{4}^{(123)}} A_{5 ; 1}^{[1 / 2]}\left(\sigma_{1}, \sigma_{2}, \sigma_{3}, \sigma_{4}, \sigma_{5}\right)= \\
A_{5 ; 1}^{[1 / 2]}(1,2,3,4,5)+A_{5 ; 1}^{[1 / 2]}(1,2,4,3,5)+ \\
A_{5 ; 1}^{[1 / 2]}(1,4,2,3,5)+A_{5 ; 1}^{[1 / 2]}(4,1,2,3,5)+ \\
\quad A_{5 ; 1}^{[1 / 2]}(3,1,2,4,5)+A_{5 ; 1}^{[1 / 2]}(3,1,4,2,5)+ \\
A_{5 ; 1}^{[1 / 2]}(3,4,1,2,5)+A_{5 ; 1}^{[1 / 2]}(4,3,1,2,5)+ \\
A_{5 ; 1}^{[1 / 2]}(2,3,1,4,5)+A_{5 ; 1}^{[1 / 2]}(2,3,4,1,5)+ \\
A_{5 ; 1}^{[1 / 2]}(2,4,3,1,5)+A_{5 ; 1}^{[1 / 2]}(4,2,3,1,5) .
\end{gathered}
$$

The partial amplitudes $A_{5 ; 1}^{[1 / 2]}(1,2,3,4,5)$ for the various helicities of the external gluons and photons are given in Ref. [11].

The extension of the Collins-Soper-Sterman soft-gluon resummation formalism [12] to diphoton production was discussed in Ref. [10]. We follow that work when calculating the $\mathcal{O}\left(\alpha_{S}\right)$ fixed-order corrections to the $q \bar{q} \rightarrow \gamma \gamma$ subprocess, including the fragmentation contributions, and resumming the contributions of the $q \bar{q} \rightarrow \gamma \gamma g, q g \rightarrow \gamma \gamma q$ and $\bar{q} g \rightarrow \gamma \gamma \bar{q}$ subprocesses. As explained in Ref. [10], the resummation of large logarithmic terms due to the emission of soft-gluons in the $g g \rightarrow \gamma \gamma g$ processes can also be completed even in the absence of the full real emission calculation, because the large logs of the type $\ln \left(Q / Q_{T}\right)$ emerge only from soft and/or collinear gluons emitted off the initial-state gluons. Thus, a prediction of the low $Q_{T}$ region for this subprocess at $\mathcal{O}\left(\alpha_{S}^{3}\right)$ precision is also possible.

In the resummation of the $g g \rightarrow \gamma \gamma g$ process, we use the $A^{(1)}$ and $A^{(2)}$ coefficients of Ref. [13], since these coefficients depend only on the initial partons and are independent of the partonic process itself. The $B^{(1)}$ coefficient for the $g g \rightarrow \gamma \gamma g$ process is the same as the one for $g g \rightarrow H g$, also given in Ref. [13]. For the $C^{(1)}$ coefficient, we use the same approximation as Ref. [10], since the basis of that approximation (both photons having large transverse momenta) is also valid here. To predict the high $Q_{T}$ region at $\mathcal{O}\left(\alpha_{S}^{3}\right)$ precision, on the other hand, requires the full calculation of $g g+q g \rightarrow \gamma \gamma X$, which was approximated in Ref. [10]. To improve the prediction of Ref. [10] for the high- $Q_{T}$ region, we make use of the $\mathcal{O}\left(\alpha_{S}^{3}\right)$ real emission calculation, described above. In the low- and mid- $Q_{T}$ region we use the resummed results of Ref. [10], matching it with the $\mathcal{O}\left(\alpha_{S}^{3}\right)$ fixed order prediction in the way which was described in Ref. [9]. The $q g$ component, which is still approximated, is highly suppressed because of the large difference between the gluon and quark luminosities in the probed region of momentum fraction.

\section{NUMERICAL RESULTS}

The above described analytic results are implemented in the ResBos Monte Carlo event generator [9]. In the numerical calculations we use the following electroweak parameters [14]:

$$
\begin{array}{rlrl}
G_{F} & =1.16639 \times 10^{-5} \mathrm{GeV}^{-2}, \quad m_{Z} & =91.187 \mathrm{GeV}, \\
m_{W} & =80.41 \mathrm{GeV}, \quad \alpha\left(m_{Z}\right)=\frac{1}{128.88} .
\end{array}
$$

We use the NLO expressions for the running electromagnetic and strong couplings $\alpha(\mu)$ and $\alpha_{S}(\mu)$, as well as the NLO parton distribution function set CTEQ4M. We set the renormalization scale equal to the factorization scale: $\mu_{R}=\mu_{F}=Q$. For the choice of the non-perturbative function of the resummation for the various subprocesses, we follow Ref. [10].

Our kinematic cuts, imposed on the final state photons, reflect the optimal detection capabilities of the ATLAS detector [4]:

- $p_{T}^{\gamma}>25 \mathrm{GeV}$, for the transverse momentum of each photon,

- $\left|y^{\gamma}\right|<2.5$, for the rapidity of each photon, and

- $p_{T}^{1} /\left(p_{T}^{1}+p_{T}^{2}\right)<0.7$, to suppress the fragmentation contribution, where $p_{T}^{1}$ is the transverse momentum of the photon with the higher $p_{T}$ value.

Additionally, we restrict the invariant mass of the photon pair in the relevant region for the light Higgs production: $80 \mathrm{GeV}<Q<150 \mathrm{GeV}$. We also apply a $\Delta R=0.4$ separation cut on the photons, but our results are insensitive to this cut (cf. Ref. [10]).

In Fig. 1 we display the invariant mass distribution of photon pairs at the LHC in the inclusive process $p p \rightarrow \gamma \gamma X$, calculated using ResBos with the above cuts. We present separately the resummed contribution from the subprocesses $\mathcal{O}\left(\alpha_{S}\right) q \bar{q}+q g \rightarrow \gamma \gamma X$ (dashed) which includes the fragmentation, and $\mathcal{O}\left(\alpha_{S}^{3}\right) g g+q g \rightarrow \gamma \gamma X$ (dotted), as well as the total distribution (upper solid), which is the sum of these two curves. We also display, for comparison, the leading order contributions from the subprocesses $\mathcal{O}\left(\alpha_{S}^{0}\right) q \bar{q} \rightarrow \gamma \gamma$ (dot-dashed) and $\mathcal{O}\left(\alpha_{S}^{2}\right)$ $g g \rightarrow \gamma \gamma$ (lower solid). We note that, although the full $\mathcal{O}\left(\alpha_{S}^{3}\right)$ corrections to the $g g \rightarrow \gamma \gamma$ process have not yet 


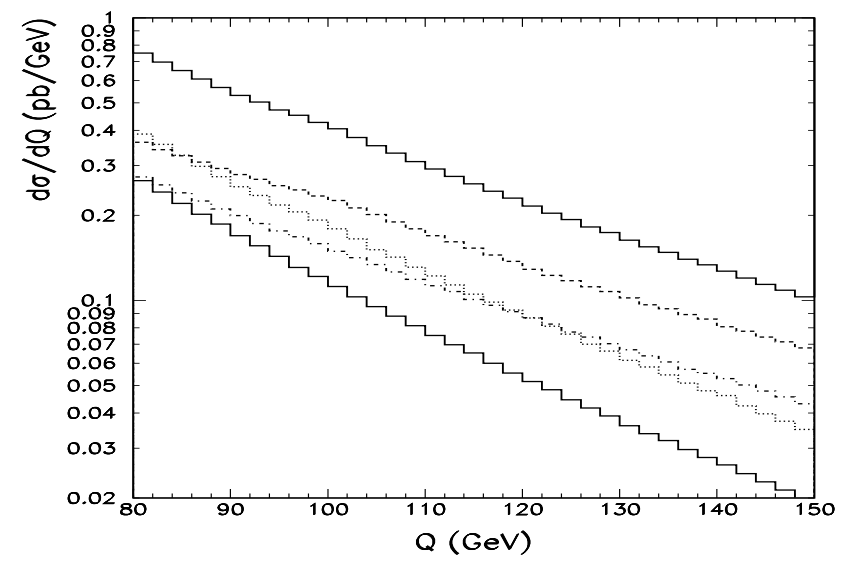

FIG. 1. Invariant mass distributions of photon pairs at the LHC, calculated using ResBos. The total curve (upper solid) is the sum of the $\mathcal{O}\left(\alpha_{S}\right) q \bar{q}+q g \rightarrow \gamma \gamma X$ (dashed) and the $\mathcal{O}\left(\alpha_{S}^{3}\right) g g+q g \rightarrow \gamma \gamma X$ (dotted) contributions. The leading order curves for the contributions from $\mathcal{O}\left(\alpha_{S}^{0}\right) q \bar{q} \rightarrow \gamma \gamma$ (dash-dotted) and $\mathcal{O}\left(\alpha_{S}^{2}\right) g g \rightarrow \gamma \gamma$ (lower solid) are also shown for comparison.

been calculated, a well-defined infrared-finite prediction for the $\mathcal{O}\left(\alpha_{S}^{3}\right) g g+q g \rightarrow \gamma \gamma X$ (dotted) contribution can be obtained by the resummation procedure, the only approximation being made in the $C^{(1)}$ coefficient. This gives a prediction for this subprocess about 1.9 times the prediction without $C^{(1)}$.

In the $Q>85 \mathrm{GeV}$ region the contribution of the $q \bar{q}+$ $q g \rightarrow \gamma \gamma X$ subprocess is higher than that of the $g g+$ $q g \rightarrow \gamma \gamma X$. From Fig. 1 we can also read the $K$-factors, which are defined as the ratios of the resummed to the leading order results. The $K$-factor for the $q \bar{q}+q g \rightarrow$ $\gamma \gamma X$ process (the ratio of the dashed and dot-dashed curves) is between 1.40 and 1.70 in the invariant mass range of interest. Similarly, the $g g+q g \rightarrow \gamma \gamma X K$ factor (the ratio of the dotted and lower solid curves) is between 1.45 and 1.75. This results in an overall $K$ factor of 1.4 to 1.7 . These $K$-factors are about the same as the $\mathrm{NLO} / \mathrm{LO} K$-factors in the fixed order perturbative calculations $[6,7]$.

In Fig. 2 we plot the transverse momentum distribution of photon pairs at the LHC. In addition to the total resummed result, we give the resummed and fixed-order calculations separately for both the $\mathcal{O}\left(\alpha_{S}\right) q \bar{q}+q g \rightarrow$ $\gamma \gamma X$ and the $\mathcal{O}\left(\alpha_{S}^{3}\right) g g+q g \rightarrow \gamma \gamma X$ subprocesses. In both cases the resummed results deviate substantially from the fixed order predictions in the $0<Q_{T}<100$ $\mathrm{GeV}$ region. At $Q_{T}=30 \mathrm{GeV}$ the resummed curves are higher by about 30 and 50 percent for the $q \bar{q}+q g \rightarrow \gamma \gamma X$ and $g g+q g \rightarrow \gamma \gamma X$ subprocesses, respectively. As a result the total resummed curve exceeds the total fixedorder prediction by almost 40 percent at $Q_{T}=30 \mathrm{GeV}$. This is the $Q_{T}$ region where the kinematic cuts are applied in order to optimize the statistical significance of the signal in the Higgs plus jet mode. Thus, the use of

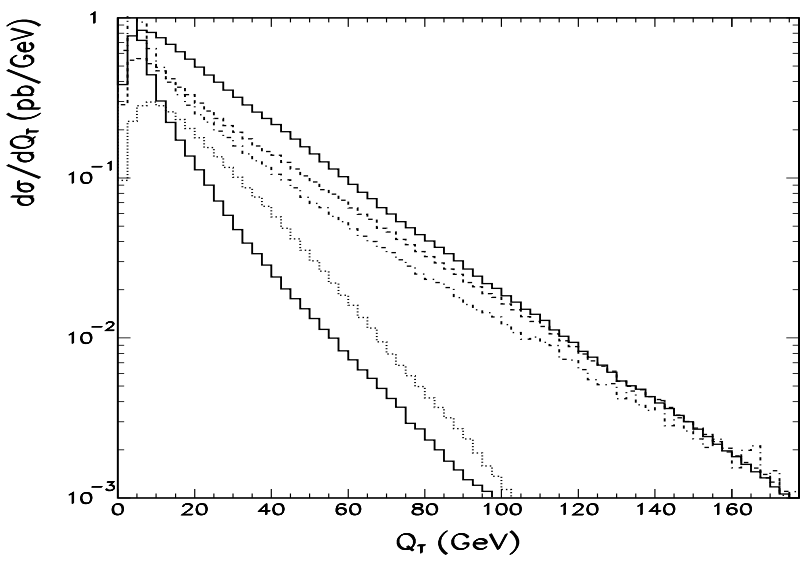

FIG. 2. Transverse momentum distributions of photon pairs at the LHC. The total resummed curve (upper solid) is the sum of the resummed $q \bar{q}+q g \rightarrow \gamma \gamma X$ (dashed), and the resummed $g g+q g \rightarrow \gamma \gamma X$ (dotted) contributions. The fixed-order curves for the contributions from $\mathcal{O}\left(\alpha_{S}\right)$ $q \bar{q}+q g \rightarrow \gamma \gamma X$ (dash-dotted) and $\mathcal{O}\left(\alpha_{S}^{3}\right) g g+q g \rightarrow \gamma \gamma X$ (lower solid) are also shown for comparison.

the resummed prediction is necessary to extract a reliable statistical significance, and also to make a correct determination of the Higgs production cross section, in the presence of kinematic cuts.

Fig. 2 also shows that if the photon pair is constrained to be in the mid- to high- $Q_{T}$ region the contribution of the $g g+q g \rightarrow \gamma \gamma X$ subprocess is small. At $Q_{T}=40$ $\mathrm{GeV}$, for example, the $g g$ initial state accounts for less than 30 percent of the total cross section. In the ResBos program, the $g g+q g \rightarrow \gamma \gamma X$ rate is predicted purely by the resummed calculation and does not cross over into the fixed-order $\mathcal{O}\left(\alpha_{S}^{3}\right)$ calculation until after about $Q_{T}=100$ $\mathrm{GeV}$, at which point this rate is negligible.

To illustrate the higher order effects on the invariant mass distribution in the presence of a $Q_{T}$ cut, in Fig. 3 we plot $Q$ of the photon pair while restricting $Q_{T}>30 \mathrm{GeV}$. Due to the different shape of the $Q_{T}$ distributions the cut offsets the fixed-order and resummed rates, as explained in Ref. [9]. The effect is larger for the $g g$ channel, since there the resummed and fixed-order $Q_{T}$ distributions deviate more, signaling higher corrections to the $g g$ process than to the $q \bar{q}$ process. In the presence of kinematic cuts the difference in the $Q$ distribution, between the fixed order and the resummed calculations, can be as high as 50 percent. The $Q_{T}>30 \mathrm{GeV}$ cut also suppresses the $g g$ channel, decreasing the uncertainty of the total prediction.

\section{CONCLUSIONS}

In this paper we present the resummed calculation of the $p p \rightarrow \gamma \gamma X$ distributions including the exact fixed 


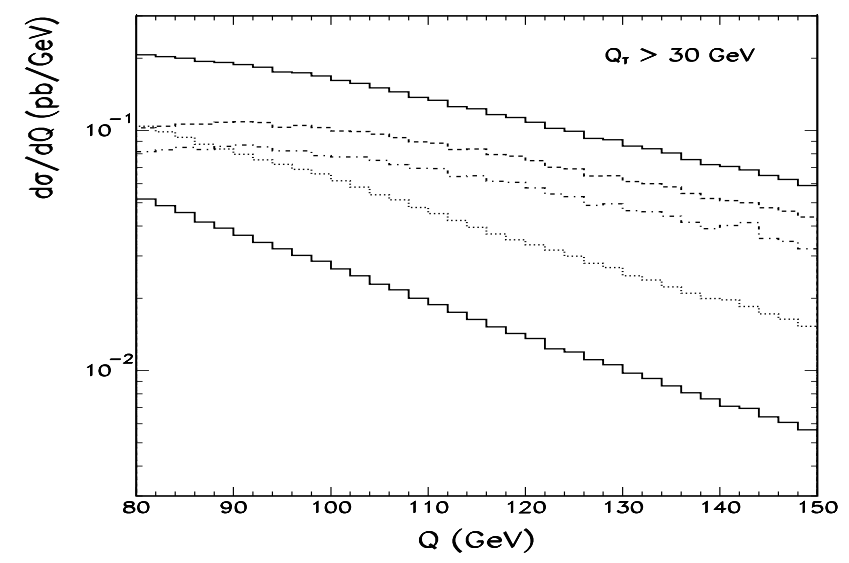

FIG. 3. Invariant mass distributions of photon pairs at the LHC, with the cut $Q_{T}>30 \mathrm{GeV}$. The total resummed curve (upper solid) is the sum of the resummed $q \bar{q}+q g \rightarrow \gamma \gamma X$ (dashed), and the resummed $g g+q g \rightarrow \gamma \gamma X$ (dotted) contributions. The fixed-order curves for the contributions from $\mathcal{O}\left(\alpha_{S}\right) q \bar{q}+q g \rightarrow \gamma \gamma X$ (dash-dotted) and $\mathcal{O}\left(\alpha_{S}^{3}\right)$ $g g+q g \rightarrow \gamma \gamma X$ (lower solid) are also shown for comparison.

order $g g \rightarrow \gamma \gamma g$ contribution. By including the most important higher order contributions, the resummed cross section provides a reliable prediction for the inclusive diphoton invariant mass and transverse momentum $\left(Q_{T}\right)$ distributions. With a $Q_{T}$ cut the least reliable $g g \rightarrow \gamma \gamma X$ component can be suppressed, and the prediction further improved.

Given our results, we propose the search for the Higgs boson in the inclusive diphoton mode with a cut on the transverse momentum of the photon pair. This measurement can be done without the requirement of a tagged jet, which is necessary in the $\gamma \gamma$ jet mode. Therefore, it is independent of the jet algorithm used, it can be performed more precisely experimentally, and it can be predicted more reliably from a resummed calculation such as presented here.

While finishing this paper we became aware of a similar work, in which the authors extract the $3 g 2 \gamma$ amplitude from the $5 g$ amplitude [15]. Our fixed order analytical and numerical results agree with the results of that paper.

\section{ACKNOWLEDGMENTS}

We thank the CTEQ Collaboration, L. Dixon and S. Mrenna for invaluable discussions, J. Huston and M. Abolins for help with the ATLAS parameters and for useful conversations. This work was supported in part by the NSF under grants PHY-9802564 and PHY-9722144.
[1] Presentations at the LEPC Physics Jamboree on March 24, 1999 by ALEPH http://alephwww.cern.ch/ALPUB/ seminar/lepc_mar99.ps, DELPHI http://delphiwww.cern. ch/delfigs/figures/vanina981112.ps.gz, L3 http://I3www .cern.ch/conferences/ps/Clare_LEPC9811.ps.gz, and OPAL http://www.cern.ch/Opal/plots/plane/lepc98.html.

[2] J. Erler and P. Langacker, hep-ph/9809352;

G. D'Agostini and G. Degrassi, hep-ph/9902226; J. Erler, hep-ph/9904235; P. Langacker, hep-ph/9905428.

[3] B. Abbott et al. (D0 Collaboration), Phys. Rev. Lett. 80, 3008 (1998); B. Abbott et al. (D0 Collaboration), hepex/9808029; F. Abe et al. (CDF Collaboration), Phys. Rev. Lett. 82, 271 (1999); W.M. Yao (CDF Collaboration), hepex/9903068; Y.K. Kim (CDF Collaboration), talk given at QCD and Weak Boson Physics Workshop, Fermilab, March 4-6, 1999, http://cdfsg6.lbl.gov/ ykkim/wmass/lathuile.ps.

[4] ATLAS Collaboration, Technical Proposal,

CERN/LHC/94-43 LHCC/P2 (1994); CMS Collaboration, Technical Proposal, CERN/LHC/94-43 LHCC/P1 (1994); CMS Collaboration, Technical Design report, CERN/LHCC/97-33 (1997).

[5] S. Abdullin, M. Dubinin, V. Ilyin, D. Kovalenko, V. Savrin, N. Stepanov, Phys. Lett. B431, 410 (1998).

[6] A. Djouadi, M. Spira and P.M. Zerwas, Phys. Lett. B 264, 440 (1991); S. Dawson, Nucl. Phys. B 359, 283 (1991); D. Graudenz, M. Spira and P.M. Zerwas, Phys. Rev. Lett. 70, 1372 (1993); M. Spira, A. Djouadi, D. Graudenz and P.M. Zerwas, Phys. Lett. B 318, 347 (1993); R.P. Kauffman and W. Schaffer, Phys. Rev. D 49, 551 (1994); S. Dawson and R.P. Kauffman, Phys. Rev. D 49, 2298 (1994); M. Spira, A. Djouadi, D. Graudenz and P.M. Zerwas, Nucl. Phys. B 453, 17 (1995).

[7] E.L. Berger, E. Braaten, R.D. Field, Nucl. Phys. B 239, 52 (1984); P. Aurenche, A. Douiri, R. Baier, M. Fontannaz, D. Schiff, Z. Phys C29, 459 (1985); B. Bailey, J.F. Owens and J. Ohnemus, Phys. Rev. D 46, 2018 (1992).

[8] C. Balázs and C.-P. Yuan, Phys. Rev. D59, 114007 (1999).

[9] C. Balázs and C.-P. Yuan, Phys. Rev. D56, 5558 (1997).

[10] C. Balázs, E.L. Berger, S. Mrenna and C.-P. Yuan, Phys. Rev. D57, 6934 (1998).

[11] Z. Bern, L. Dixon, D. Kosower, Phys. Rev. Lett. 70, 2677 (1993).

[12] J.C. Collins, D. Soper, Nucl. Phys. B193, 381 (1981); Nucl. Phys. B213, 545(E) (1983); Nucl. Phys. B197, 446 (1982); J.C. Collins, D. Soper, G. Sterman Phys. Lett. B109, 388 (1982); Nucl. Phys. B223, 381 (1983); Phys. Lett. B126, 275 (1983); J.C. Collins, D. Soper, G. Sterman, Nucl. Phys. B250, 199 (1985).

[13] C.-P. Yuan, Phys. Lett. B283, 395 (1992); R.P. Kauffman, Phys. Rev. D45, 1512 (1992).

[14] Particle Data Group (C. Caso et al.), "Review of Particle Physics", The European Physical Journal C 3, 1 (1998).

[15] D. de Florian, Z. Kunszt, preprint ETH-TH-99-15, hepph/9905283. 